\section{Plant genomics roundup}

The Tomato Genome Consortium's sequence of the tomato genome provides insights into the genetic basis for fruit texture and ripening times. In maize, a secondgeneration haplotype map will help guide future breeding efforts, and the sequencing of 278 inbred strains sheds light on the genetic effects of past breeding efforts. Finally, the gene encoding a novel cytochrome P450, CYP76AD1, has been identified as the one responsible for red betalain production in beets, a strong antioxidant and commercially important pigment.

The Tomato Genome Consortium. The tomato genome sequence provides insights into fleshy fruit evolution. Nature 485, 635-641 (2012).

Chia, J.M. et al. Maize HapMap2 identifies extant variation from a genome in flux. Nat. Genet. advance online publication, doi:10.1038/ng.2313 (3 June 2012).

Jiao, Y. et al. Genome-wide genetic changes during modern breeding of maize. Nat. Genet. advance online publication, doi:10.1038/ng.2312 (3 June 2012).

Hatlestad, G.J. et al. The beet $R$ locus encodes a new cytochrome P450 required for red betalain production. Nat. Genet. advance online publication, doi:10.1038/ng.2297 (3 June 2012).

\section{CNVs from exome sequencing}

Numerous large clinical cohorts comprising thousands of individuals are being analyzed by exome sequencing. Until now, exome sequencing data analysis has yielded primarily single-nucleotide variants, but in a recent issue of Genome Research, Krumm et al. describe a new algorithm that allows the identification copy number variants from these data sets. Their approach makes use of singular-value decomposition to remove noise resulting from both nonuniformity in exome capture reactions and systematic biases between batches of samples. The authors benchmark their method on two sets of samples for which both exome and single-nucleotide polymorphism (SNP) microarray data were available to call copy number variants. On eight HapMap samples, exome analysis identified $>68 \%$ of the 32 variants called by microarray approaches, whereas on data from a cohort of autism spectrum disorder patients, $94 \%$ of the 124 calls made by the authors' method were concordant with results from either array analysis or quantitative PCR. This method could be applied to several recently published data sets, such as a study of DNA sequence variants in drug target genes (Science advance online publication, doi:10.1126/science.1217876, 17 May 2012) or the exomes of 2,440 individuals of European and African ancestry (Science advance online publication, doi:10.1126/science.1219240, 17 May 2012; Genome Research advance online publication, doi:10.1101/ gr.138115.112, 14 May 2012).

\section{Keeping gut bugs from causing harm}

The human gut is colonized by commensal bacteria that are essential for normal development and metabolism. In healthy individuals, these gut bugs inhabit the intestinal lumen, the epithelial surface and gut-associated lymphoid tissues, but in numerous chronic diseases, including Crohn's

Written by Laura DeFrancesco, Susan Jones, Jason Kreisberg \& Craig Mak disease, they stray into surrounding tissues and cause inflammation. Inflammation is regulated by interleukin 22 (IL-22), which is produced by immune cells, including innate lymphoid cells (ILCs), a newly identified family of cells that seem to bridge the innate and adaptive immune systems in mammals. Sonnenberg et al. report that IL-22-producing ILCs are present in human, non-human primate and mouse intestinal samples. Antibody-mediated depletion of ILCs in mice resulted in bacteria (mainly Alcaligenes species) disseminating to the spleen and liver, with a concomitant increase in signs of inflammation. Case-control studies revealing Alcaligenes spp. in sera of pediatric Crohn's disease patients and cirrhotic hepatitis $\mathrm{C}$ virus-infected individuals confirm that loss of containment of gut bacteria is clinically relevant. Crucially, administering IL-22 in mice ameliorated the effects of ILC depletion and kept the gut bugs in their place. Taken together, these data support a model in which ILCs function to confine commensals to specific niches where they cannot cause harm and could pave the way to rational strategies to treat selected inflammatory diseases. (Science 336, 1321-1325, 2012)

SJ

\section{siRNAs on the edge}

The systematic use of therapeutic short-interfering RNAs (siRNAs) is limited by their very short half-life in the blood and their lack of tumortargeting capabilities. Although packaging siRNAs into nanoparticles, such as liposomes or cationic polymers, can mitigate some of these shortcomings, it is difficult to produce a homogenous collection of nanoparticles using these approaches. Lee et al. now report a new type of DNA-based nanoparticle for siRNA delivery. These self-assembling nanoparticles are built from six different DNA oligonucleotides, resulting in a uniform population of tetrahedron-shaped particles where each of the six edges can accommodate one siRNA molecule. To increase the tumor-targeting capabilities, the authors conjugated folic acid onto the siRNAs. These triangular pyramids are designed to be large enough to minimize uptake by the kidneys but small enough to enter solid tumors through the leaky vasculature. Upon intravenous injection into a tumorbearing mouse, fluorescently labeled tetrahedrons containing folic acid conjugated-siRNA were primarily localized to the tumor and the kidney. Serum half-life of nanoparticle-borne siRNA was increased significantly over naked siRNA. Systemic injection knocked down targeted gene expression in the tumor. Future studies will explore the potential to use these and other types of DNA-based nanoparticles to deliver other therapeutic agents. (Nat. Nanotechnol. 7, 389-393, 2012)

$J K$

\section{Mending hearts in situ}

The adult heart is incapable of healing after heart attacks or other insults to the heart muscle. Instead, scar tissue typically forms around the damaged area from proliferating cardiac fibroblasts, which outnumber cardiac myocytes by about two to one. Two groups have demonstrated a way to shift the balance toward myocytes by transfecting cardiac fibroblasts in situ with a set of transcription factors that direct heart development. And more incredibly, in a mouse model of heart attack, the induced cardiomyocytes improve cardiac function, despite the fact that only a few percent of cells in the area are transfected. The factors involved (GATA4, MEF2C, TBX5 and HAND2) had previously been shown to transdifferentiate fibroblasts to myocytes in vitro. Now by injecting retroviruses carrying three or four of the factors in or around the area of damage, fibroblasts take on the appearance and the expression patterns of myocytes. As the recovery seems out of line with the efficiency of transfection, other elements, such as growth factors or cytokines, perhaps attracted to the site, may be involved. (Nature 485, 593-598, 2012; Nature 485, 599-604, 2012) 